\title{
Epidemiology of Creutzfeldt-Jakob Disease in Floridian Men and Women: 1988-2012
}

\author{
Robert B Hood ${ }^{1}$, Richard B Early ${ }^{2}$, Zachary Weber ${ }^{1}$, Judith A Schwartzbaum ${ }^{1}$, Sharona B Ross ${ }^{2}$, \\ Alexander S Rosemurgy II ${ }^{2}$ and Randall E Harris ${ }^{1,3 *}$ \\ ${ }^{1}$ The Ohio State University, College of Public Health, USA
}

${ }^{2}$ Department of General Surgery, Florida Hospital, USA

${ }^{3}$ The Ohio State University, College of Medicine, USA

*Corresponding author: Randall E Harris, The Ohio State University, College of Public Health, Columbus, Ohio, College of Medicine, USA.

To Cite This Article: Randall E Harris, Epidemiology of Creutzfeldt-Jakob Disease in Floridian Men and Women: 1988-2012. Am J Biomed Sci \& Res. 2019 - 6(4). AJBSR.MS.ID.001050. DOI: 10.34297/AJBSR.2019.06.001050.

Received: 㠿 November 18, 2019; Published: 眥 December 02, 2019

\begin{abstract}
Creutzfeldt-Jakob disease (CJD) is a rare and fatal prion brain disorder with unknown etiology. In the current study, we characterized the epidemiology of CJD in the population of Florida. Cases of CJD were identified using the principal diagnoses by ICD-9 code among 55 million inpatient hospital discharge records reported to the Florida Center for Health Information and Transparency from 1988 to 2012 . We examined basic demographic and clinical characteristics, and trends in the annual incidence for men and women. We identified 390 patients with CJD. Most patients were Caucasian $(90.0 \%$ ) and $51.3 \%$ were male. The median age of hospitalization was 67 years (Interquartile range: 14). The overall annual incidence was 0.91 per million (95\% Confidence Interval: $0.8-1.0)$ and the overall rate was slightly higher for men than women (0.94 versus 0.87 per million, respectively). Annual rates were similar for men and women except for the period from 2001 to 2010 when the rates diverged sharply. Among cases, $12.3 \%$ died in the hospital and $23.1 \%$ were discharged to hospice. CJD cases identified through hospital records in Florida have similar characteristics to previously reported data. Case-control studies are needed to elucidate environmental triggers of this rare condition.
\end{abstract}

Keywords: Creutzfeldt-Jakob Disease; Epidemiology; Hospitalizations; Florida

Abbreviations: CJD: Creutzfeldt-Jakob disease.

\section{Introduction}

Creutzfeldt-Jakob disease (CJD) is a rare degenerative brain disorder that is uniformly fatal. CJD is characterized by prion protein misfolding leading to accumulation of amyloid fibrils in the central nervous system and neurodegeneration [1,2]. Hallmark features of CJD are neuronal vacuolation, neuronal death, and pronounced glial reactions with rapidly progressive dementia, seizures, myoclonus, ataxia and eventually the absence of voluntary movements and speech/akinetic mutism [3].

Four subtypes of CJD exist:

1. Variant,

2. Iatrogenic,

3. Genetic,

4. Sporadic [2].
Variant CJD (vCJD) is associated with consuming meat products tainted with bovine spongiform encephalopathy (BSE) [4]. Additionally, vCJD has been shown to be transmissible through blood transfusions [5,6]. Iatrogenic CJD (iCJD) are cases that arise due to medical procedures [7]. Although the evidence is far from definitive, there are reports of iCJD following neurosurgery, transplantation of contaminated corneas or administration of contaminated human pituitary hormones [7]. Sporadic CJD (sCJD) is by far the most common form of the disease accounting for 85$90 \%$ of all cases [8,9]. Genetic CJD (gCJD) occurs in families in an autosomal dominate fashion and accounts for $10 \%$ of all CJD cases [8]. vCJD and iCJD have earlier age of onset typically in the 3rd and 4th decade while sCJD and gCJD occur typically in the 5th and 6th decade of life. Rare cases of sCJD during adolescence have also been reported [3]. 
CJD is difficult to diagnose due to symptom variability and similarity to other diseases associated with dementia [10]. CJD can only be definitively diagnosed with a brain biopsy or autopsy. However, the cerebrospinal fluid along with electroencephalography can provide a probable diagnosis of CJD [10]. Even with diagnostic challenges, reports on the incidence on CJD ranges from 1 to 2 cases per million per year [8,9,11-13]. The epidemiology of CJD has been described using surveillance studies and large national registries. Study limitations include incomplete reporting and lack of autopsies for definitive diagnosis. We examined the epidemiology of CJD in the population of Florida from 1988 to 2012. We demonstrate that cases identified through inpatient hospital discharge records provide similar epidemiologic profiles as those cases identified at autopsy or through disease specific registries. To describe the epidemiology of CJD in Florida, we characterize demographic and clinical characteristics and estimate gender-specific annual incidence rates and trends in the rates for a 25 -year time period.

\section{Materials and Methods}

We obtained data from the Florida Center for Health Information and Transparency (FCHIT). The FCHIT is a branch of Florida's Agency for Health Care Administration and is tasked with collecting discharge data from all hospitals in Florida including acute care, psychiatric and rehabilitation hospitals among other types. The dataset contained over 55 million records of de-identified inpatient hospital discharge records for all hospitals located in Florida from 1988 to 2012. We identified cases of CJD using the principal diagnosis coded in ICD-9 format (046.10-046.19). Cases included a mix of both residents and non-residents of Florida.

We complied basic demographic characteristics including age, sex, race, ethnicity and resident status and tested for differences in medians and frequencies between Florida and non-Florida cases using Mann-Whitney tests and Chi square tests. Due to small sample size, we collapsed race into three categories; white, black and other races which included Asian, American Indian or Alaskan Naïve. We obtained frequencies for clinical characteristics of cases examined included admission type, admitting diagnosis (patients after 2005 only), length of stay, status at discharge, principal procedures and secondary diagnosis codes. To obtain annual age-adjusted incidence rates of CJD, only cases who were permanent residents of Florida were utilized. We age-adjusted incidence rates using the 2000 U.S. Census. We examined incidence rates separately for men and women as well as across genders. Trends were examined using 3-year moving averages of the Florida incidence rates. Other descriptive characteristics were based upon combined data for both Florida and non-Florida cases. Statistical analyses were conducted using SAS version 9.4 (SAS Institute, Cary N.C.).

\section{Results and Discussion}

A total of 390 cases of CJD were identified from the Florida inpatient hospital records during 1988-2012. Most cases ( $\mathrm{n}=369$ ) were Florida residents (Table 1). Distributions of age, gender and ethnicity were similar for Florida and non-Florida cases. Four patients did not have resident information and were excluded from these comparisons. There were slightly more men $(n=200)$ than women ( $\mathrm{n}=190)$ and the vast majority of cases $(90.0 \%)$ were white. Most cases $(92.1 \%)$ were diagnosed after 50 years of age. The median onset in women was earlier than men (65 versus 69 years of age, $\mathrm{P}<0.05$ ). Only $12.3 \%$ of cases died in hospital whereas $53.6 \%$ were discharged either to Hospice or Homecare. Patients had a median hospital stay of approximately 9 days (range: 0 - 144 days). The most common comorbidities reported were hypertension (38.2\%), urinary tract infection (22.1\%), hyperlipidemia (14.4\%), diabetes (10.3\%) and hypothyroidism (9.5\%).

\begin{tabular}{|c|c|c|c|}
\hline Characteristic & & $\mathbf{n}$ & $\%$ \\
\hline \multirow{2}{*}{ Sex } & Female & 190 & $48.70 \%$ \\
\hline & Male & 200 & $51.30 \%$ \\
\hline \multirow{5}{*}{ Age } & $<49$ & 31 & $7.90 \%$ \\
\hline & $50-59$ & 63 & $16.20 \%$ \\
\hline & $60-69$ & 136 & $34.90 \%$ \\
\hline & $70-79$ & 117 & $30.00 \%$ \\
\hline & $80+$ & 43 & $11.00 \%$ \\
\hline \multirow{4}{*}{ Race $†$} & White & 305 & $89.70 \%$ \\
\hline & Black & 19 & $5.60 \%$ \\
\hline & Other & 16 & $4.70 \%$ \\
\hline & Missing & 50 & \\
\hline \multirow{3}{*}{ Ethnicity } & Hispanic & 39 & $11.50 \%$ \\
\hline & Non-Hispanic & 299 & $88.50 \%$ \\
\hline & Missing & 52 & \\
\hline
\end{tabular}




\begin{tabular}{|c|c|c|c|}
\hline \multirow{2}{*}{ Resident status $\ddagger$} & Florida & 369 & $95.60 \%$ \\
\hline & Other & 17 & $4.40 \%$ \\
\hline \multirow{3}{*}{ Admission type* } & Emergency & 198 & $50.80 \%$ \\
\hline & Urgent & 113 & $29.00 \%$ \\
\hline & Other & 79 & $20.20 \%$ \\
\hline \multirow{4}{*}{ Discharge status• } & Dead & 48 & $12.30 \%$ \\
\hline & Homecare & 119 & $30.50 \%$ \\
\hline & Hospice & 90 & $23.10 \%$ \\
\hline & Other & 133 & $34.10 \%$ \\
\hline \multirow{2}{*}{ Length of stay (in days) } & & Median & Range \\
\hline & & 9 & 0. -144 \\
\hline
\end{tabular}

† Other race includes all other races besides white and black.

$\ddagger$ Other includes residents of states other than Florida.

* Other admission type are admissions not covered by the categories reported (i.e., Elective, Newborn, Other).

- Other discharge status includes discharge to short-term hospitals, skilled nursing, intermediate care facilities, Medicare facilities, psychiatric hospitals, and other institutions.

The overall age-adjusted incidence rate of CJD was 0.91 cases per million persons (95\% Confidence Interval [CI]: 0.8, 1.0). Men had a slightly higher age adjusted incidence than women $(0.94$ cases per million persons versus 0.87 cases per million). We observed high variability in the annual age-adjusted incidence rates across the 25-year time period (Figure 1). Interestingly, the rates for men and women ran in close parallel except for the period 2001 to 2010, during which there was significant divergence with men having a peak incidence of 1.49 cases per million compared to women having a nadir of 0.33 cases per million $(\mathrm{P}<0.01)$. Among 55 million inpatient hospital discharge records reported for Florida residents during 1988 to 2012, we found 369 cases whose principal diagnosis was CJD with an additional 21 cases from non-Florida residents. Using the census data for this period, the annual ageadjusted incidence of CJD in the Florida population was 0.91 per million persons during 1988 to 2012. The male rate was slightly higher than the female rate $(0.94$ cases versus 0.87 cases per million persons).

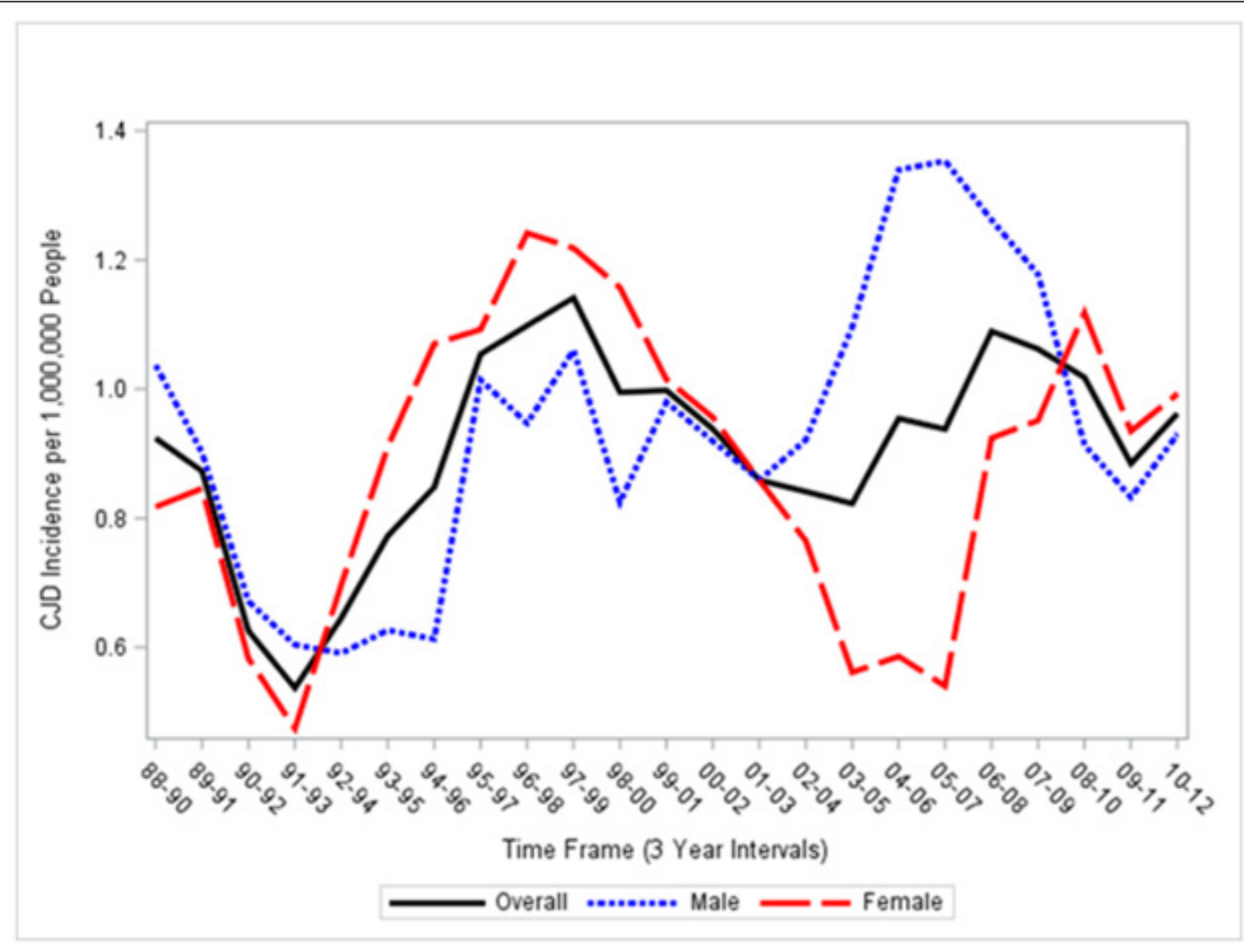

Figure 1: Three Year Moving Averages of Annual Incidence Rates of Creutzfeldt-Jakob per Million in Florida, 1988-2012 [1]. 
Annual incidence rates of CJD varied widely across the 25year study period potentially reflecting fluctuation in unknown environmental exposures. Annual rates for men and women were similar except for a sharp divergence during 2001-2010 in which the peak rate for men was 4.5 times higher than the nadir rate for women (1.49 versus 0.33 cases per million, $\mathrm{P}<0.01$ ). This abrupt divergence in the male and female rates may have been caused by the introduction of a gender-specific environmental or iatrogenic factor leading to the acute onset of excess male cases of sCJD. Only 3 $(0.8 \%)$ of the 390 cases of CJD were diagnosed before age 30 years. It is probable that these younger cases were genetic forms of CJD. However, we cannot confirm this due to a lack of specificity of the ICD-9 codes reported in the hospital records.

Prior investigations have found results similar to our hospital sample [9,11-13]. For example, the annual incidence rate from CJD in the United States estimated from death certificate data yielded a rate of 1 per million persons [12]. Furthermore, demographic and clinical characteristics of the 390 Florida CJD cases were similar to prior surveillance studies of CJD [11]. However, unlike previous studies, we were unable to assess the frequency of specific symptoms of the disease or the subtype of CJD [11]. We did however collect information on the prevalence of common secondary diagnoses in these patients that included hypertension, urinary tract infection, hyperlipidemia, type 2 diabetes, and hypothyroidism. Whether any of these comorbid conditions are involved in the pathogenesis of CJD is a topic for future investigation.

Several limitations were inherent in these Florida hospital data. These included the level of missing data and the lack of information on the temporality of comorbid events. As a consequence, estimates of the prevalence of comorbid conditions are likely lower than actual values and their potential role(s) in pathogenesis could not be determined. In addition, certain clinical characteristics and diagnostic features were not uniformly reported until later years thereby preventing consideration of CJD subtypes in the analysis. Such information would have facilitated a more detailed examination of the divergent patterns observed in men and women. Additionally, it is possible that a single patient could have had multiple inpatient visits. However, because CJD is a rapid and fatal disease it unlikely that multiple hospitals visits occurred especially with a majority of cases either dying in hospital or being discharged to hospice care. Notwithstanding these limitations, the Florida CJD sample had demographic and clinical characteristics similar to that reported in surveillance-based studies, and it is not likely that these shortcomings biased our findings. However, cautious interpretation is advisable and clearly, well designed epidemiological studies are needed to further clarify the etiology of CJD.

\section{Conclusion}

Cases of CJD identified through hospital records in Florida have similar characteristics to those in other studies. Overall, the annual rate was 0.91 per million but rates fluctuated widely during the 25-year study period with divergent patterns for men and women from 2001 to 2008. Well-designed case control studies are needed to elucidate environmental triggers of this rare condition.

\section{Acknowledgments}

We thank all members of the Florida Center for Health Information and Transparency (FCHIT), a branch of Florida's Agency for Health Care Administration, for their services in the collection and computerization of hospital discharge data from all hospitals in Florida during the study period.

\section{Conflicts of Interest}

None to declare.

\section{References}

1. Brown K, Mastrianni JA (2010) The prion diseases. J Geriatr Psychiatry Neurol 23(4): 277-298.

2. Aguzzi A (2006) Prion diseases of humans and farm animals: epidemiology, genetics, and pathogenesis. J Neurochem 97(6): 17261739.

3. Appleby BS, Appleby KK, Rabins PV (2007) Does the presentation of Creutzfeldt-Jakob disease vary by age or presumed etiology? A metaanalysis of the past 10 years. J Neuropsychiatry Clin Neurosci 19(4): 428-435.

4. Chen CC, Wang YH, Wu KY (2013) Consumption of bovine spongiform encephalopathy (BSE) contaminated beef and the risk of variant Creutzfeldt-Jakob disease. Risk Anal 33(11): 1958-1968.

5. Lefrere JJ, Hewitt P (2009) From mad cows to sensible blood transfusion: the risk of prion transmission by labile blood components in the United Kingdom and in France. Transfusion 49(4): 797-812.

6. Gregori L, Yang H, Anderson S (2011) Estimation of variant CreutzfeldtJakob disease infectivity titers in human blood. Transfusion 51(12): 2596-2602.

7. Hamaguchi T, Noguchi Shinohara M, Nozaki I, Nakamura Y, Sato T, et al. (2009) The risk of iatrogenic Creutzfeldt-Jakob disease through medical and surgical procedures. Neuropathology 29(5): 625-631.

8. Chen C, Dong XP (2016) Epidemiological characteristics of human prion diseases. Infect Dis Poverty 5(1): 47.

9. Brandel JP, Peckeu L, Haik S (2013) The French surveillance network of Creutzfeldt-Jakob disease. Epidemiological data in France and worldwide. Transfus Clin Biol 20(4): 395-397.

10. Caobelli F, Cobelli M, Pizzocaro C, Pavia M, Magnaldi S, et al. (2015) The role of neuroimaging in evaluating patients affected by CreutzfeldtJakob disease: a systematic review of the literature. J Neuroimaging 25(1): 2-13.

11. Gubbels S, Bacci S, Laursen H, Hogenhaven H, Cowan S, et al. (2012) Description and analysis of 12 years of surveillance for CreutzfeldtJakob disease in Denmark, 1997 to 2008. Euro Surveill 17(15).

12. Holman RC, Belay ED, Christensen KY, Maddox RA, Minino AM, et al. (2010) Human prion diseases in the United States. PLoS One 5(1): e8521.

13. Litzroth A, Cras P, De Vil B, Quoilin S (2015) Overview and evaluation of 15 years of Creutzfeldt-Jakob disease surveillance in Belgium, 19982012. BMC Neurol 15: 250. 